\title{
Onomastique et parodie dans la saga bédéïque Crepúsculon
}

\author{
Onomastic and parody in the Crepúsculon cartoon saga
}

\author{
Sonia Fournet-Pérot \\ Université de Limoges, France
}

\begin{abstract}
Résumé : Le mythe du vampire a inspiré de nombreuses créations littéraires et audio-visuelles qui ont, pour certaines, cherché à atteindre un public adolescent. C'est le cas de la saga cinématographique Twilight (adaptation des romans de Stephenie Meyer) que les quatre BD espagnoles du cycle Crepúsculon s'emploient à parodier. Nous aborderons Crepúsculon en adoptant une approche exclusivement linguistique, et, en particulier, dans le cadre de cet article, onomastique, par le biais des tropes rhétoriques.
\end{abstract}

Mots-clés : onomastique, parodie, BD espagnole, linguistique, tropes rhétoriques.

\begin{abstract}
The myth of the vampire inspired many literary and audio-visual creations. Some of them tried to seduce a young audience. This is the case of the Twilight saga films (adaptation of the Stephenie Meyer novels), that the four Spanish comics of the cycle Crepúsculon want to parody. We will study Crepúsculon adopting an exclusively linguistic approach and more precisely, in this article, an onomastic approach, through rhetorical tropes.
\end{abstract}

Keywords: onomastics, parody, Spanish comics, linguistics, rhetorical tropes.

\section{Introduction}

Le mythe du vampire, cet être duel, qui survit en absorbant la vie, dont la cruauté n'a d'égal que l'amour tourmenté et dont on se plaît à exposer et explorer la supposée noirceur, a inspiré de nombreuses créations littéraires et audio-visuelles qui ont connu, ces dernières années, un renouveau spectaculaire, et qui ont cherché pour certaines à étendre leur réception à un public adolescent, avec, dans un premier temps, la série Buffy (1997) qui a ouvert la voie à - parmi les plus célèbres: Angel (1999), Vampire High (2001), Being Human (2008 pour la version britannique), Vampires diaries (2009, adaptation d'une série littéraire à succès débutée en 1991) et surtout à la saga cinématographique Twilight, adaptation des romans de Stephenie Meyer et dont le premier opus date de 2008. Cette dernière production tend particulièrement à édulcorer la monstruosité vampirique afin de l'ajuster à la tranche d'âge visée, transformant le revenant «rarement anim[é] de bonnes intentions » décrit par Claude Lecouteux (2004:7) en un potentiel héros bienveillant, capable de délaisser toute velléité diabolique et d'avoir pour seuls guides ses sentiments, amicaux, fraternels et surtout amoureux. Ce postulat implique une réécriture doucereuse du mythe, que notre corpus, formé des quatre BD du 
cycle Crepúsculon (2009, 2010, 2011 et 2012'), s'emploie à mettre en lumière. Les scénaristes Pepe Caldelas et Ferrán Toro parodient ouvertement - c'est leur déclaration d'intention dès la première de couverture ${ }^{2}$ - ce qu'ils considèrent déjà, selon nous, comme étant déjà une parodie. Nous en voulons pour preuve la mutation subie par la première phrase du texte original, que l'on entend au tout début du film en voix off : «Nunca me había detenido a pensar en cómo iba a morir $1^{3}$, laquelle met l'accent sur le côté prétendument dramatique de l'histoire - la mort, dont les vampires sont traditionnellement la personnification, étant présentée comme la finalité du récit -, et qui devient, sous la plume des bédéistes : « Nunca me había planteado cómo me iba a enamorar "), reformulation qui insiste sur la nature sentimentale de l'œuvre de Meyer et de son adaptation cinématographique, le pouvoir de séduction du vampire l'emportant, selon eux, sur sa duplicité démoniaque et mortifère. Pour exhumer cette critique sous-jacente - et afin de coller au plus près à notre sujet - nous aborderons Crepúsculon d'une manière intentionnellement monstrueuse en laissant de côté toute dimension iconographique et en adoptant une approche exclusivement linguistique, et, en particulier, dans le cadre de cet article, onomastique, chaque toponyme et anthroponyme nourrissant la visée parodique, ludique mais aussi critique de l'œuvre source.

\section{Le vampire vampirisé : un substrat mythique disqualifié}

L'énonciation d'un nom propre est un acte de «référence dénominative », pour reprendre la terminologie de Georges Kleiber, par le biais duquel une instruction cognitive est envoyée à l'interlocuteur quant à la recherche d'un référent portant le nom en question. Apparaissent ainsi dans la BD des références dénominatives qui, bien qu'absentes du texte source et ne renvoyant à aucun des protagonistes, sont loin d'être anodines, puisqu'elles s'enracinent dans le terreau audio-visuel formant le contexte, au sens sperbérien (Sperber \& Wilson, 1989) du terme, spécifique de l'expert en revenants à dents longues. Le prénom "Lestat ", cité Tome 1, p. 6 est un clin d'œil évident au personnage créé par Anne Rice en 1976 dans ses Chroniques de vampires, dont le premier opus sera porté à l'écran en 1994 par Neil Jordan (Entretien avec un vampire) et le troisième en 2002 par Michael Rymer (La reine des damnés). La protagoniste, "Isabella ", surnommée "Bella ", voit son patronyme "Swan " remplacé par "Lugosi " (Tome 2 : 40), référence explicite au nom de scène de Blaskó Béla Ferenc Dezső, l'acteur hongroaméricain qui a interprété le Comte Dracula en 1931 dans le film éponyme de Tod Browning ${ }^{4}$. Le lycée, enfin, point névralgique de la trame de Twilight, se nomme "True Blood» (Tome $1: 3$ ), titre d'une série télévisée réalisée par Alan Ball et inspirée du cycle La communauté du Sud, de Charlaine Harris, dont la diffusion a débuté en 2008. Ces trois citations onomastiques connotent des représentations du vampire très éloignées de celles données à voir dans l'adaptation des romans de Meyer : adultes, cruels, pouvant être dotés de sentiments mais ne manifestant aucun respect pour la vie humaine, et, pour les plus récents, ambivalents sexuellement et

\footnotetext{
1 Par souci de clarté, nous parlerons plus avant des tomes 1, 2, 3 et 4, correspondant respectivement aux ouvrages édités en 2009, 2010, 2011 et 2012.

2 Est en effet précisé, en bas de la première de couverture du Tome 1 : «El Cómic que parodia a la exitosa película que adapta oficialmente a la aclamada novela. ॥.

3 " l'd never given much thought to how I would die [...] " (Meyer, 2005 : Preface).

4 Il fut essentiellement cantonné par la suite aux rôles de vampires : Tim Burton lui rendit hommage dans Ed Wood en 1995.
} 
profondément libertins, de nombreuses scènes de True Blood notamment étant réservées à un public averti. Ce décalage référentiel, manifeste pour le lecteur éclairé, est explicité linguistiquement par le cotexte. Le vampire d'Anne Ryce est ainsi mentionné dans une exclamation invocatrice : "por los colmillos de Lestat», qui opère une focalisation réductrice sur les canines du vampire, le vidant de toute profondeur et qui, dans le même temps, prend toute sa saveur lorsque l'on sait que les suceurs de sang de Twilight sont tous dépourvus de ces crocs affutés et sont donc à la fois superficiels et incomplets, lacunaires. La série érotico-gore True Blood est également mentionnée, accolée au nom «Institute »: on nous raconte bien une histoire de vampires... pour lycéens! La graphie est susceptible à elle seule de retranscrire cet écart avec le substrat mythique convoqué : «Béla Lugosi » devient "Bella », l'altération orthographique étant le miroir de la dégradation mythique, que Caldelas et Toro critiquent ostensiblement dès le premier tome : la spécialité de la maison - de la maison Twilight, s'entend - c'est la "Kankamusa » (p. 13), à savoir l'imposture, le leurre, l'illusion. Non seulement la BD nous met en garde contre les romans de Meyer et leur adaptation cinématographique qui nous trompent sur la marchandise, mais la nature même de la modification orthographique de " cancamusa » ancre résolument l'histoire dans le monde adolescent.

\section{Un vampire en pleine crise... d'adolescence}

L'utilisation de la graphie «k », bien qu'absente de l'alphabet espagnol traditionnel, est en effet habituelle dans le langage sms et internet prisé par les nouvelles générations pour retranscrire l'occlusive vélaire sourde. Le sociolecte juvénile se distingue également par son goût pour l'emphase, comme le remarque Luisa Montero Curiel :

[l]os jóvenes, especialmente efusivos en su forma de hablar y de actuar, utilizan en su conversación una gran cantidad de expresiones en las que el sentido superlativo desempeña un papel fundamental (2011:89).

Au sein de notre corpus, la transformation des titres du premier et du dernier tome de la saga : Crepúsculo et Amanecer, en Crepúsculon et Madrugón donne immédiatement le ton. Ce suffixe augmentatif " -ón " vient également pervertir le patronyme maintes fois répété de la famille de vampires au centre de l'intrigue, les gentils "Cullen » devenant les "Culon ", l'avancement de l'accent pouvant correspondre à une réécriture volontairement américanisante (Lapesa, 1999 : 535602) ou à un désir de conserver une similitude non seulement consonantique mais aussi tonique avec le terme source. Nous reviendrons plus avant sur les enjeux sémantiques de cette transformation suffixale qui revient à assigner aux principaux protagonistes un nom signifiant littéralement " gros culs ». L'utilisation de diminutifs hypocoristiques - "Rafa " et "Pili " (originellement les vampires "Laurent " et "Victoria ») - vient renforcer la familiarité du langage employé, en totale discordance avec la nature des personnages désignés qui sèment la mort dont ils sont la matérialisation. Quand l'onomastique ne renvoie pas au monde adolescent par le registre choisi, elle s'y insère naturellement en raison des réalités évoquées,

- parfois directement : «Bella »-qui, rappelons-le, est également un diminutif pourrait connoter le personnage de La Bella y la Bestia de Disney, allusion potentielle que les bédéistes s'empressent d'investir dès la page 2 du tome 1 ; 
- parfois par substitution : «Royce King Jr», le fiancé de Rosalie Cullen dans le texte source, devient «Sir Jonas Brother », groupe de pop rock américain pour adolescents ;

- parfois par création: le nom des méchants vampires, les "Volturis", est remplacé par le mot-valise ${ }^{5}$ "Teleturis» (Tome 2:36), qui renvoie manifestement à son paronyme «Teletubis», série télévisée pour enfants.

Ces croisements linguistiques - graphiques, suffixaux, lexicaux - mêlent le sang de deux entités inconciliables : la bête séculaire assassine au langage suranné et froid rencontre l'adolescent adepte de dessins animés et de boys bands et dont l'immaturité et les affects polluent l'expression. Cette corruption adolescente du mythe n'est ni sporadique ni anodine, comme en témoigne l'accumulation de télescopages destructeurs mis en mots par les auteurs de notre corpus, dont le titre du film que Bella et l'une de ses amies vont voir au cinéma offre une synthèse éloquente: "Larry Poter y la piedra secreta del cáliz de Askaban del prícipe fénix ॥ (Tome $2: 11$ ). Caldelas et Toro cherchent à mettre l'accent sur les lourdeurs, les approximations, les erreurs et les incohérences du discours source et ils y parviennent également en massacrant les anthroponymes d'origine des personnages, de sorte qu'ils ne se contentent pas d'évoquer un substrat mythique ou adolescent, mais qu'animés par la folie créatrice des auteurs, ils transcendent leur statut de noms propres pour signifier bien plus qu'une simple référence dénominative.

\section{L'onomastique ou le reflet du vampire : quand le nom se fait rature 6}

D'un point de vue pragmatique, il existerait trois types de référence: indicative, descriptive et dénominative. Les noms propres ne véhiculent pas de référence indicative, puisqu'ils ne nécessitent pas la présence du référent, ni de référence descriptive dans la mesure où ils ne décrivent rien. Cependant, en 1981, Kleiber affirmait dans Problèmes de référence. Descriptions définies et noms propres, p. 325, que les noms propres ne pouvaient être dénués de sens, sans quoi ils ne permettraient pas d'effectuer un acte de référence. En 1995, il précisera que les noms propres ont ainsi un sens de dénomination à la fois instructionnel et descriptif : instructionnel car ils enjoignent à rechercher le référent dénommé ; descriptif, car si "le nom propre ne décrit pas le particulier qu'il désigne », il dénomme malgré tout un particulier (Kleiber, $1995: 11-36)$. C'est par rapport à ces derniers points que les anthroponymes de notre corpus opèrent une première transgression, car ils ne se limitent pas à dénommer un particulier, mais le décrivent systématiquement - au sens propre du terme -, en mettant en exergue un trait perçu comme définitoire. L'onomastique de Crepúsculon est en ce sens incisivement métonymique et profondément caricaturale, chaque personnage se voyant réduit à la spécificité exposée par son patronyme. Chaque nom propre est ainsi retravaillé par les bédéistes qui privilégient quatre types de procédés re-créatifs et récréatifs: l'antonomase, le mot-valise - que nous avons déjà évoqué -, les figures de répétition phonétiques et le calembour.

\footnotetext{
5 "Création verbale formée par le télescopage de deux (ou trois) mots existant dans la langue» (Centre National de Ressources Textuelles et Lexicales, disponible sur [http://www.cnrtl.fr/definition/motvalise]).

${ }^{6}$ Ce titre est un calembour, mauvais, comme il se doit.
} 


\subsection{Antonomases}

L'antonomase est un trope qui consiste à employer un nom propre comme un nom commun, ou, plus rarement, un nom commun comme un nom propre, cas de figure que nous rencontrons précisément dans notre corpus. L'antonomase du nom commun peut adopter une formulation outrancière en étant construite sur une périphrase, ainsi qu'en attestent les exemples suivants : « - ¿Nombre? - iijUn gilipollas con una furgoneta!!! » (Tome $1: 10$ ) ; «Esto... Señor indio que sale en el cómic sólo para que lo maten... ¿̇sabe por dónde anda Luke? " (Tome 2 : 18). "Tyler Crowley " n'est plus qu'un couillon avec une fourgonnette et c'est bien là son seul rôle dans I'histoire : fournir, par la non-maîtrise de son véhicule, un prétexte à la découverte de la véritable nature du vampire. Son vrai nom est accessoire, car son identité, dénuée de profondeur et stigmatisée par le recours au lexique grossier, l'est tout autant; seule compte son action ponctuelle, synthétisée par la fourgonnette. La dénomination d'u Harry Clearwater ", I'Indien en sursis, ne fait que confirmer cette hypothèse : le personnage est une coquille vide, un simple outil dont la personnalité est inutile à l'intrigue. L'antonomase la plus courante consiste à remplacer un anthroponyme par un nom commun ; souvent qualifiée d'antonomase d'excellence, elle « marque donc [...] la perfection d'une personne dans un domaine donné " (Leroy, 2001 : 27). Notre support textuel recourt à cette figure pour requalifier la tribu des "Quileutes ॥, peuple amérindien de l'état de Washington, que Meyer réinvente sous les traits d'hommes-loups, ennemis héréditaires des vampires, et dont l'histoire fondatrice est celle du chef "Taha Aki ॥ et de sa troisième épouse, qui se sacrifiera pour sauver la lignée lycanthrope et dont, pourtant, nous ignorons le nom. Qu'à cela ne tienne, Caldelas et Toro se chargent de combler cette lacune conformément à leur perception de cette inclusion monstrueuse : les «Quileutes» deviennent les «Tajaos». Deux remarques s'imposent : d'un point de vue phonétique, la syncope de l'occlusive dentale sonore en position intervocalique (tajados > tajaos) est la marque d'une langue orale, d'une familiarité que vient confirmer le signifié de " tajados", qui renvoie à " tajada », terme trivial que l'on pourrait traduire, en respectant le registre relâché, par " cuite, biture ». Le peuple prétendument mythique a ici un goût prononcé pour l'alcool, spécificité martelée par la requalification de ses principaux représentants en noms de cocktails populaires: "Calimocho » et sa 37ème épouse, "Mojito ", l'hyperbole venant renforcer la caricature d'une société fictive dont la légende imaginée par l'auteure de la saga est, pour les bédéistes, une histoire à dormir debout et dont les actes et les propos ne peuvent résulter que d'un éthylisme excessif. L'antonomase présente donc les métamorphes lupins comme de parfaits ivrognes, l'exposition de l'excellence dont elle est le support étant manifestement dépréciative, accentuant corrélativement le décalage caricatural. Nous ajouterons à ces exemples une occurrence un peu particulière, puisque l'antonomase est le fruit de la création d'un mot valise. Dans Twilight, l'histoire d'amour entre Bella et Edward Cullen se solde par un mariage et la naissance d'un enfant, une petite fille mihumaine, mi-vampire, que Bella baptise "Renesmée " en unissant simplement, quoiqu'étrangement, les prénoms de sa mère - "Renée " - et de sa belle-mère - "Esmée ». Dans la parodie espagnole, Bella utilise la première syllabe du prénom de chacune de ses trois tantes : "Bernarda ", "Zoraida " et "Tasiana ", ce qui donne "Berzotas» (Tome 4:34), substantif familier désignant une personne ignorante ou idiote, soit, littéralement, une "andoville »! La créativité attribuée à l'héroïne est 
ridiculisée et, dans le même temps, le fruit de l'union des deux protagonistes principaux est présenté comme une ineptie, une absurdité de plus. De la même façon qu'on ne peut agglutiner aléatoirement des prénoms pour n'en former qu'un seul, une humaine et un vampire ne peuvent procréer. La fillette est un autre accroc au mythe qui veut que ces créatures soient stériles, et sa dénomination dans la BD permet, une fois encore, de souligner l'écart grandissant entre la représentation traditionnelle du vampire et sa réécriture par Meyer. Remarquons également que si "Renesmée " était phonétiquement composé de l'intégralité des prénoms constitutifs, «Berzotas » n'est constitué que de fragments de prénoms et se voit donc réduit à un agglomérat d'identités incomplètes, reflétant l'imperfection d'un univers fantastique en décomposition. Cet appauvrissement sémantique et référentiel, traduit linguistiquement par un appauvrissement morphologique, apparaît également lors de la production de certains anthroponymes à partir de figures de répétition.

\subsection{Paréchèses et oméotéleutes}

La paréchèse est la "répétition vicieuse de la même syllabe " (Barre, 1839 : 753), elle concerne exclusivement le groupe de vampires ennemi de la famille Cullen dans le premier tome, à savoir, "James ", "Victoria " et "Laurent ", renommés respectivement "Rubén Rubiales ", " Pili Pelirroja " et "Rafa Rastas ॥. Ces prénoms ont été choisis afin de s'accorder phonétiquement avec des patronymes tenant plus du surnom que du nom en raison de leur caractère intrinsèquement descriptifs puisqu'ils se réfèrent à la chevelure des morts vivants concernés. James est blond (" rubio »), Victoria est rousse (" pelirroja ») et Laurent, rasta (" rastas ») ; or c'est à ces considérations capillaires, et donc superficielles, que sont limités ces personnages une fois parodiés. Deux d'entre eux, nous l'avons vu, sont même affublés de diminutifs, la réduction morphologique traduisant leur insignifiance diégétique mais aussi leur personnalité inconsistante, leur incomplétude. Le prénom du meneur - «Rubén »-n'est pas tronqué, l'extension linguistique soulignant à la fois son rôle de leader et de potentiel assassin de Bella. Outre ces considérations référentielles et morphologiques, les paréchèses, en frôlant la cacophonie, tendent à ridiculiser les personnages en tournant en dérision leurs dénominations qui, étant proches du bégaiement ou du vire-langue, transforment les noms de meurtriers en puissance en jeux phonétiques enfantins, d'autant plus mauvais qu'ils peuvent être approximatifs (" Pili ॥ \# "Peli »), tout comme les vampires qu'ils désignent ne sont que des approximations risibles du mythe.

L'oméotéleute, qui met en scène la répétition d'une terminaison identique, n'est appliquée qu'à l'homme-loup qui participe au triangle amoureux au centre de l'œuvre de Meyer: «Jacob Black», qui devient, dans le tome 2, « Jacobo el lobo », prénom espagnolisé suivi d'un surnom identificateur. L'homéotéleute, en créant une rime interne, permet elle aussi de se railler d'un personnage à travers l'adjectif qui se forme naturellement, par association de signifiants, dans l'esprit de l'énonciataire : « Jacobo » + «lobo »= «bobo », et de fait il est vrai que les bédéistes appuient particulièrement sur la carence neuronale du jeune homme. Paréchèses et homéotéleutes mettent ainsi en évidence une dissonance phonique qui fait de nos âmes traditionnellement damnées des êtres aux contours maladroits, clownesques. La disqualification des personnages passe également par des figures plus complexes, jouant à la fois sur la phonétique, la syntaxe et la sémantique. 


\subsection{Calembours}

Selon Luque Durán, il s'agit d'un « juego del lenguaje que se produce cuando las sílabas de una o varias palabras contiguas son agrupadas de una forma distinta a la usual " (2007 : 97). Le calembour consiste donc en une resegmentation du discours, elle-même fondée sur une homophonie syntaxico-discursive. Dans notre corpus, cette homonymie est construite sur des groupes de mots (Prénom + Nom) et donne naissance à ce que Guiraud nomme "calembour "segmenté" " (Guiraud, 1976 : 12), lequel peut-être approximatif, auquel cas on parle d'à-peu-près. Autre particularité: la structure des calembours diffère en fonction du groupe de personnages concerné : les loups d'un côté, les vampires de l'autre.

Les dénominations choisies pour les loups soulignent leur nature de métamorphes ${ }^{7}$, le secret inviolable devenant secret de polichinelle et les grosses ficelles de la trame étant, par là même, mises à nu. Jakob Black, le lycanthrope amoureux éconduit, mais tenace, de Bella, est ainsi présenté, dès la p. 2 du tome 1, - et avant de se rebaptiser "Jacobo el lobo" - sous le nom de "Luke Antropo ", calembour segmenté phonétiquement approximatif du substantif " licántropo ". En d'autres termes, dès les premières pages de la parodie, non seulement le suspense, à peine installé, est réduit à néant, mais l'à-peu-près phonique met en évidence le fait que la figure meyerienne du loup-garou ne sera qu'une turlupinade. En témoignent les patronymes du reste de la meute. L'Alpha se nomme "Sam Bernardo " et les autres membres, "Rod Bailer ", "Hernando Berman " et "Felipe Quinés ». Le chef de bande se distingue par le calembour phonétiquement parfait dont il fait l'objet, la graphie " -m " traduisant le rapprochement naturel du point d'articulation de la nasale alvéolaire [n] de celui de l'occlusive bilabiale sonore [b] qui la suit immédiatement. Les autres sont beaucoup plus boiteux, soit par une correspondance phonique fragmentaire ("Rod Bailer " \# "Rottweiler ॥), soit par une correspondance morphologique fragmentaire (" Hernando Berman $) \rightarrow$ " dobermán »; " Felipe Quinés » $\rightarrow$ " pequinés »). Si les à-peu-près témoignent de la monstruosité défectueuse des personnages, le sémantisme convoqué met encore plus à mal leur lycanthropie. Hormis pour "Luke Antropo ", seul membre de la meute au premier plan de l'intrigue, l'onomastique transforme les hommes-loups sanguinaires en hommes-chiens, marquant la dévaluation de l'animal sauvage une fois métamorphosé en son cousin apprivoisé. Eł quels canidés ! Si le Rottweiler et le Doberman restent des chiens de garde, le Saint Bernard, initialement dressé pour le secours en montagne - c'est d'ailleurs lui qui retrouvera Bella, égarée dans les bois -, est associé à un caractère affectueux et maladroit depuis le succès du film grand public Beethoven en 1992, - bien que Cujo, adapté de Stephen King en 1983, ait pu nuancer cette perception chez les adeptes du genre horrifique. E† que dire du Pékinois ? Le grand méchant loup fait place à l'épagneul nain... En renommant de la sorte la tribu lupine de Twilight, les bédéistes brocardent la vision qu'en donne Meyer, la bête maléfique légendaire étant, à leur sens, tenue en laisse, défaite, avilie, ridiculisée.

Leurs ennemis de toujours ne sont pas épargnés par les calembours, mais le jeu est mené différemment, pour bien des raisons. Premièrement, il n'est pas explicité. On nous annonce le nom des personnages, puis, plus loin, leur prénom : « Por ahí vienen

\footnotetext{
7 |l en va de même pour « Jacobo el lobo ».
} 
los Culon. Esos son Rósandol y Métel. [...] Ésa es Ándade y el chico que parece tan tenso es Tóntol. ॥ (Tome $1: 4$ ) Cela requiert une lecture active, le lecteur devant se faire lectant pour recomposer et saisir des calembours qui ne lui sont plus servis sur un plateau. L'altération du patronyme est déjà en elle-même un indice. Comme nous l'avons précédemment souligné, " culón » signifie, selon la Real Academia Española (RAE), « [q] ue tiene muy abultadas las posaderas ", guidant le regard du lecteur vers une partie de l'anatomie fortement sexualisée ; c'est donc sur l'apparence séduisante et séductrice des buveurs de sang que l'on est amené à se focaliser, mais à l'excès, comme pourrait en témoigner le suffixe augmentatif. Dans le même temps, l'angle de vue choisi par les scénaristes de Crepúsculon est humiliant : non seulement le physique l'emporte sur l'intellect, mais il est réduit à sa parcelle la plus indélicate, jetant à terre la prestance, l'élégance, le raffinement habituellement associés aux vampires. Le terme «culón»est par ailleurs homonymique, puisqu'il désigne également familièrement, selon la RAE, un soldat invalide. Bien que ce soit le premier signifié qui soit activé à la lecture des prénoms que nous analyserons plus avant - cette seconde acception nous semble éloquente: le prédateur avide de sang et dont les capacités physiques font un combattant hors pair est présenté, par le biais du nom de famille que lui assignent les bédéistes, comme un éclopé. II n'est donc plus apte à remplir sa fonction première et devient un simulacre de vampire. Le parallèle avec l'œuvre parodiée est flagrant : dans Twilight, les « gentils » vampires boivent du sang, mais uniquement du sang non humain et juste pour survivre, certes pas pour s'amuser, refoulant ce qui fait l'essence même du mythe ! Deuxièmement, les prénoms des protagonistes sont des prénoms imaginaires : "Rósandol », "Métel », "Ándade " et "Tóntol », ce qui est non seulement un nouvel indice quant à la présence d'un éventuel jeu de mots onomastique, mais peut-être également un moyen d'illustrer linguistiquement l'invraisemblance des personnages imaginés par Meyer. Troisièmement, une fois que l'on a recomposé ces monstres de Frankenstein dénominatifs démembrés, les calembours plus ou moins approximatifs qui prennent forme ne dévoilent pas, cette fois, la nature monstrueuse - ici vampirique - des personnages, mais s'attardent, vulgairement, sur des considérations beaucoup plus triviales exhibées comme signe distinctif des enfants Cullen. Dans la saga littéraire et cinématographique, ces derniers se présentent aux humains comme formant une fratrie, alors que, hormis Edward, ils sont en couple : Rosalie et Emmett d'un côté, Alice et Jasper de l'autre. À chacun de ces duos correspondent des calembours s'appuyant sur des champs sémantiques distincts. C'est ainsi le sexe qui unit le premier couple : "Rósandol Culon » et "Métel Culon » sont des à-peu-près de «rozándo(le) el culo » et " métele en el culo ", syntagmes faisant respectivement étalage, en termes peu châtiés, de palpations fessières et de sodomie. Pour le second duo, le substantif "culo " n'est plus une métonymie libidineuse mais permet d'exprimer un jugement de valeur dépréciatif visant à discréditer leur intelligence : "Ándade Culon » et «Tóntol Culon» constituent des calembours de "anda de culo» et "tonto del culo». C'est l'association d'une paumée écervelée (qui, par ailleurs, est censée voir l'avenir alors que " de culo » signifie, selon la RAE, " hacia atrás») et de l'idiot du village. Bref, les premiers ne pensent qu'à forniquer et les seconds sont décérébrés, ces portraits on ne peut moins flatteurs étant encore dévalorisés par la présence de l'augmentatif et la grossièreté des dénominations qui n'est que le pendant de la grossièreté de ces personnages, mal finis, imparfaits, approximatifs. 


\section{En guise de conclusion}

Une première conclusion s'impose: pour toutes ces transformations et re-créations onomastiques, le degré de recherche et de difficulté des jeux de mots croît proportionnellement à l'importance des personnages dans la diégèse du texte source, l'élaboration des calembours pouvant donner lieu, notamment, à une multiplicité d'inférences interprétatives. En d'autres termes, plus Meyer a accordé de place au ludé (référents du texte source), plus les bédéistes se sont employés à façonner un ludant8 (l'onomastique du texte transformé) complexe, ludant par ailleurs toujours réducteur - l'espace Prénom + Nom étant déjà en lui-même très restreint -, dégradant et, par conséquent, caricatural.

Cette caricature est le fruit d'une volonté parodique des bédéistes, qui mettent en scène le conflit entre des visions antagonistes du mythe vampirique et, à plus petite échelle, du mythe lupin. La parodie littéraire, rappelons-le, serait, selon TranGervat:

[...] la réécriture ludique d'un système littéraire reconnaissable (†exte, style, stéréotype, norme générique...), exhibé et transformé de manière à produire un contraste comique, avec une distance ironique ou critique (2006).

Il y aurait donc bivocalité, pour reprendre la terminologie bakhtinienne, et même ici bivocalité dissonante dans la mesure où le discours de l'auteur - Caldelas et Toro - ne se confond pas avec celui de ses personnages, deux intentions distinctes s'affrontant : « celle - directe - du personnage qui parle, et celle - réfractée - de l'auteur $॥$ (Bakhtine, 2003 : 144), ce à travers une reconstruction avec transformations d'un texte source, toujours perceptible, que nous nommerons Contexte 1 (Twilght). Le Contexte 2 (Crepúsculon) - résultat de cette reconstruction - est donc à la fois une captation - puisqu'il permet sa reconnaissance - et une subversion du Contexte 1. Cette subversion est précisément mise en lumière par le biais des transformations onomastiques qui cherchent à exhiber l'insignifiance, le manque de relief, l'absurdité, la superficialité des personnages du Contexte 1. Cette dévalorisation n'est possible que parce qu'elle se fonde sur l'existence d'un Contexte 0 , sous-jacent, celui du mythe classique, auquel il est fait référence à plusieurs reprises et dont les auteurs de notre corpus n'ont de cesse de dénoncer l'exsanguination impitoyable infligée par les romans de Meyer et leur adaptation cinématographique. Le Contexte 2 réécrit ainsi sur un mode humoristique, parce que caricatural, le Contexte 1, afin de mettre au pilori la réécriture, jugée dégradante, grossière, fautive d'un Contexte 0 de référence.

L'onomastique, «arène [de] la lutte »-pour reprendre les termes de Bakhtine (1970: 267) - entre les voix discordantes de la parodie, nous donne ainsi à voir des vampires de nature bien différente qui semblent sortir de cercueils gigognes : le plus petit, le vampire outrancier de la BD, fait couler la grenadine, arrache des dents

\footnotetext{
8 « Pour qu'il y ait jeu de mots, deux conditions sont nécessaires :

a. l'existence d'une structure duale qui motive une lecture double ;

b. la présence d'un jeu au niveau de l'encodage et du décodage assuré par un ensemble de transformations qui ménage des invariants permettant de reconstituer les deux pendants de tout jeu de mots : le ludé ou l'unité à l'état initial, avant de recevoir une manipulation ludique [...] et le ludant ou l'unité transformée sous l'effet du jeu linguistique [...] ") (Ben Amor, $2004: 209$ ).
} 
postiches et offre un bain de soleil au vampire diaphane de Twilight - qui, sous la plume de Meyer, n'a même pas la décence de brûler, soit dit en passant -, lequel avait poignardé en plein cœur le vampire légendaire. Par bonheur, le pieu était en carton-pâte...

\section{Bibliographie}

\section{Corpus}

Caldelas, P., Toro, F., Cifuentes, V. \& Arreola, U. (2009). Crepúsculon. Barcelona : Panini Comics.

- (2010). La Saga Crepúsculon. Luna lunera. Barcelona : Panini Comics.

Caldelas, P., Toro, F., Cifuentes, V. \& Ritter, K. (2011). La Saga Crepúsculon. Eclipsados. Barcelona : Panini Comics.

Caldelas, P., Toro, F., Cifuentes, V. \& Garcia Cruz, D. (2012). La Saga Crepúsculon. Madrugón. Barcelona: Panini Comics.

MEYER, S. (2005). Twilight. London: Hachette Digital.

\section{Études}

BaKhtine, M. (1970). La Poétique de Dostoïevski. Paris : Seuil.

BAKHTINE, M. (2003). Esthétique et théorie du roman. Paris : Gallimard.

BARRE, L. (1839). Complément du Dictionnaire de l'Académie française. Bruxelles: Société typographique belge Adolphe Wahlen et Cie.

BEN AMOR, T. (2004). Polylexicalité, polysémie et jeu de mots. Syntaxe et sémantique, 5, pp. 207-222.

GUIRAUD, P. (1976). Les jeux de mots. Vendôme: PUF.

Kleiber, G. (1981). Problèmes de référence. Descriptions définies et noms propres. Paris: Klincksieck.

Kleiber, G. (1995). Sur la définition des noms propres: une dizaine d'années après. In NoAllly, M. (ed.), Nom propre et nomination. Paris : Klincksieck, pp. 11-36.

LAPESA, R. (1999). Historia de la lengua española. Madrid: Gredos.

LeCOUteuX, C. (2004). Histoire de vampires : autopsie d'un mythe. Paris : Imago.

LEROY, S. (2001). Entre identification et catégorisation, l'antonomase du nom propre en français (Thèse doctorale). Université Paul Valéry - Montpellier III.

LUQUe DuRAN, J. DE D. (2007). Los juegos lingüísticos :fallos comunicacionales, humorismo verbal y reflexión metalingüística. In LUQUE TORO, L. (ed.), Actas del I Congreso internacional de léxico español actual. Venecia-Treviso: Università Ca' Foscari Venezia, pp. 91-126. 
MONTEIRO CURIEL, L. (2011). Mola mogollón : la superlación morfológica y léxica en el lenguaje juvenil. Revista de Estudios de Juventud, 93, pp. 89-104.

Sperber, D. \& Wilson, D. (1989). La Pertinence. Communication et cognition. Paris : Minuit.

TRAN-Gervat, Y.-M. (2006). Pour une définition opérationnelle de la parodie littéraire: parcours critique et enjeux d'un corpus spécifique. Cahiers de Narratologie, 13. http://journals.openedition.org/narratologie/372 [09/03/2020]. https://doi.org/10.4000/narratologie.372 